\title{
El empoderamiento de la mujer en las luchas sociales por el derecho a vivir en la ciudad. Caso CODECO
}

\section{The empowerment of women in social struggles for the right to live in the city. CODECO case}

\author{
María Graciela Ayala de Villalba ${ }^{\circledR}$, José Albino Soto Fernández ${ }^{1}{ }^{\oplus}$, Jessica \\ Graciela Castiñeira Giménez ${ }^{1}$, Mercedes Ríos $^{1}{ }^{\oplus}$, Rosa $_{\text {Ruffinelli }}{ }^{\circledR}$
}

\begin{abstract}
Resumen
En la presente investigación se describe el empoderamiento de la mujer en las luchas sociales por el derecho a vivir en la Ciudad, identifica la participación de las mujeres en la organización comunitaria e identificar las prácticas y vivencias de liderazgo en la organización. Como técnica de investigación se utilizó la entrevista en profundidad a una lideresa de la organización a partir de una guía de preguntas abiertas con la finalidad de responder a los objetivos de la investigación. La recolección de datos se realizó a partir de la visita realizada a la organización como parte de la cátedra Abordaje profesional II, Área mujer, de la carrera de Trabajo Social. Para la construcción del mismo se trabajó conceptos como las movilizaciones, participaciones y luchas de las mujeres por los derechos a una vivienda, una mejor calidad de vida y las reivindicaciones de las mujeres organizadas del bañado norte desde CODECO sobre sus territorios. Como resultado se observa las desigualdades sociales que existen, como se da y caracteriza el empoderamiento de las mujeres en dichas luchas sociales, la participación activa de mujeres organizadas para el cumplimiento de los derechos y mejoramiento de la calidad de vida de la población.
\end{abstract}

Palabras clave: empoderamiento, luchas sociales, organización comunitaria, participación.

\begin{abstract}
This research describes the empowerment of women in social struggles for the right to live in the City, identify the participation of women in community organization and identify leadership practices and experiences in the organization. As a research technique, an indepth interview with a leader of the organization was used from a guide of open questions in order to respond to the objectives of the research. The data collection was carried out from the visit made to the organization as part of the Professorship Approach II, Women's Area, of the Social Work career. For its construction, concepts such as the mobilizations, participation and struggles of women for the rights to housing, a better quality of life and the demands of the organized women of the northern bathed from CODECO
\end{abstract}

\author{
1 Universidad Nacional de \\ Asunción, Facultad de Ciencias \\ Sociales. San Lorenzo, Paraguay. \\ Correspondencia: \\ gracielita801505@gmail.com \\ Recibido: \\ 28 de setiembre de 2021 \\ Aceptado: \\ 4 de noviembre de 2021 \\ Doi: \\ https://doi.org/10.54549/ky.6e.2021.32
}

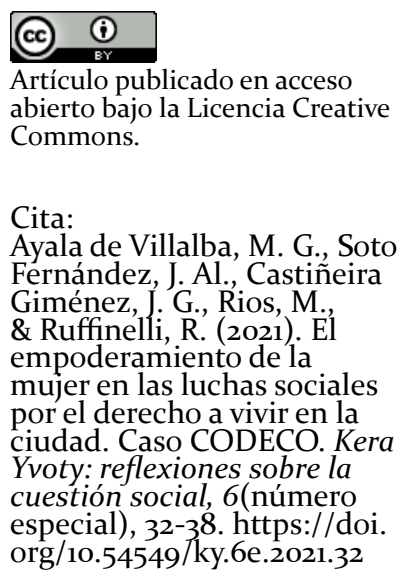


over their territories were worked on. As a result, it is observed the social inequalities that exist, how the empowerment of women in these social struggles occurs and is characterized, the active participation of women organized for the fulfillment of rights and improvement of the quality of life of the population.

Keywords: empowerment, social struggles, community organization, participation.

\section{Introducción}

Para el Paraguay la corrupción que dejó el gobierno de Stroessner bajo el poder totalitario adecuado a censuras y monopolios de los medios de comunicación, la usurpación de las tierras y el uso discrecional, se apoderó de cada sector con riquezas que se mantenía en la minoría, afectando así significativamente la economía del país, colonizando tierras que ya tenían dueños pero que estos por ser pobres y campesinos no podían hacer nada al respecto, eran acechados, torturados y sus derechos eran solo una fachada de un gobierno con poder militar.

Es así como actualmente todas las expresiones de la cuestión social no solo provienen del capitalismo sino también de estas prácticas totalitarias de tiempos pasados. Si bien el país adopta un Estado de democracia, aún existen sectores con pensamientos muy conservadores, con una historia que marcó el presente y tras varias luchas sociales, reivindicaciones y esfuerzos diarios, se pretende un futuro diferente.

No solo en Latinoamérica sino también en gran parte del mundo, existen grupos sociales que tienen esa disputa y luchas constante de clases contra el sistema patriarcal, capitalista y por sobre todo dictatorial que existió y sigue presente actualmente, estos movimientos sociales revelan sus fuerzas contra todas las desigualdades y represiones, feministas como Kate Millet, quien junto a otras mujeres, revolucionaron la teoría política al analizar las relaciones de poder que estructuran la familia, la sexualidad y sintetiza en la frase "lo personal es político", la propuesta del movimiento feminista de politizar el espacio privado: aquello que el pensamiento social y político patriarcal había designado como ámbito de la naturaleza (Pleyers, 2018). Touraine (2000) señala que los movimientos sociales "constituyen intentos fundados en un conjunto de valores compartidos para redefinir las formas de acción social e influir en sus consecuencias" (p. 101). Mediante estos movimientos de mujeres que se van formando es importante replantearse, analizar y sobre todo ver los conceptos que se tienen para un cambio, donde se visibilice la diversidad, desde la identidad de género, desde otras dimensiones como la clase, la etnicidad/racialidad, el ciclo vital, la preferencia e identidad sexual, el derecho a una vivienda en la ciudad, entre otras.

Castells (1995) afirma que los movimientos no son esenciales, no los rige una "direccionalidad predeterminada" y, por tanto, "no hay movimientos sociales 'malos' ni 'buenos'. Todos son síntomas de nuestras sociedades y chocan con las estructuras sociales, con intensidades variables y resultados que deben establecerse mediante la investigación" (p. 93).

Es importante señalar que actualmente el Poder Ejecutivo cuenta con una Secretaría de la Mujer. Estas son algunas de las diferencias importantes con otros movimientos sociales en Paraguay, como el de trabajadores. (...) Actualmente el movimiento de mujeres está integrado por una serie de nucleaciones, las cuales se diferencian entre sí por el énfasis en algunos aspectos relacionados a la discriminación y en el tipo de organización en el cual se encuentran articuladas, así como también por su mayor o menor grado de simpatía y acercamiento 
a planteamiento de tipo feminista. (Palau, 1997. p. 15)

La forma de organización de las mismas también es sumamente variada, por un lado, están aquellas que se conforman dentro de organizaciones mixtas como un sindicato o una asociación de profesionales-, por otro, aquellas integradas exclusivamente por mujeres, las cuales a su vez pueden incorporar o no elementos de género dentro de sus reivindicaciones. (Palau, 1997) ahora bien, las demandas de los movimientos de mujeres van desde reivindicaciones específicas de un sector determinado, como la valoración del trabajo doméstico planteado por la Asociación de Amas de Casa, hasta reivindicaciones más amplias como mayores espacios de participación planteada por la Comisión de Mujeres de un sindicato, de una organización campesina o de un partido político (Palau, 1997).

Tal como señalan Heikel y Peroni (1996) citados en Palau (1997) las mujeres tuvieron un estilo combinado de participación: foros previos en las cuales se movilizaron y participaron las mujeres, la formación de una Interbancada de Mujeres y la presencia constante de una ONG de mujeres, que se había propuesto como objetivo central "lograr que la Constitución paraguaya contenga las disposiciones y normas fundamentales para garantizar la plena vigencia de la democracia en Paraguay sin discriminación de ningún tipo" (p. 16).

Elmovimientodemujeresen Paraguay presenta una serie de particularidades: no tiene la estructuración clásica de un movimiento social, su accionar está condicionado a elementos externos más que a una dinámica propia, está compuesto por una serie de pequeñas organizaciones con instancias de coordinación más que de dirección, a pesar de estas particularidades, que en algunos casos pueden ser vistas como limitaciones, ha logrado importantes conquistas en el campo de la legislación durante estos últimos años (Palau, 1997).

Al mencionar sobre las luchas por las tierras se señala en primer lugar lo estipulado por la ley por el cual se señala lo siguiente; Todos los habitantes de la República de Paraguay tienen derecho a una vivienda digna. El artículo 100 de la Constitución de la República del Paraguay dice que "el estado establece las condiciones para hacer efectivo este derecho $y$ promoverá planes de vivienda de interés social, especialmente destinados a familias de escasos recursos, mediante sistemas de financiamientos adecuados". A pesar de lo estipulado en la constitución muchos paraguayos y paraguayas deben enfrentar luchas por la tierra, en un país donde unos pocos tienen mucho y muchos no tienen lo básico para vivir dignamente como seres humanos, porque el sistema favorece a esos pocos para que sigan acumulando a costa de los más desfavorecidos.

En esta tarea el desafío que tiene la CODECO desde sus inicios es la defensa comunitaria como bandera principal y en eso se basa su fundamento original, busca articular las organizaciones de base donde se busca la titulación de las tierras y defender el derecho a vivir en la ciudad.

En la organización no se mira desde el enfoque existencialista sino en el marco de los derechos en la lucha por las tenencias de tierras, la tarea es capacitar a los pobres, tocar el tema de la violencia contra las mujeres, cuesta, pero se hace énfasis para la visibilidad, ya sea en la municipalidad, MOPC, para que mucha población siga estando en sus respectivos territorios, con luchas organizativas que se da a través de articulaciones. (Co- Bañado, Articulación Sur).

A CODECO lo que lo define es su lucha por la tierra, es lo que la origina y para lo cual se constituye la organización. Lleva adelante esta labor por medio de ramas que hacen capacitaciones y microemprendimientos, encabezados por mujeres, pues ellas son las que siempre estuvieron para defender este derecho, y en 
este proceso se fueron formando a partir de sus propias vivencias y dificultades, que es lo que se busca escribir en esta investigación ¿Cómo se da el empoderamiento de la mujer en las luchas sociales por el derecho a vivir en la Ciudad?

\section{Metodología}

La investigación planteada es de tipo descriptivo, de carácter cualitativo. La fuente de información es primaria. El objetivo general de la investigación es describir el empoderamiento de la mujer en las luchas sociales por el derecho a vivir en la Ciudad, y los específicos son identificar la participación de las mujeres en la organización comunitaria e identificar las prácticas y vivencias de liderazgo en la organización. Como técnica de investigación se utilizó la entrevista en profundidad a una lideresa de la organización a partir de una guía de preguntas abiertas a fin de responder a los objetivos de la investigación. La recolección de datos se realizó a partir de la visita realizada a la organización como parte de la cátedra Abordaje profesional II, Área mujer, en el mes de febrero del año 2021.

\section{Resultados y Discusiones}

CODECO forma parte de la plataforma de organizaciones sociales, por ende, es una organización comunitaria por la lucha del derecho a vivir en la ciudad y al derecho de ser libres, no solo como personas de derechos. La lucha de CODECO y de las mujeres organizadas de este movimiento siempre fueron la defensa del territorio y la defensa de sus comunidades.

CODECO está conformada por 10 comunidades hace aproximadamente más de 40 años, comenzó como el Co- Bañado y con el paso del tiempo fue evolucionando en base a las necesidades de la comunidad.

hubo mucha necesidad, después de un tiempo personas de la organización se separaron de la organización, con todos esos cambios existentes se vio la necesidad de volver a cambiar el nombre, se empezó a trabajar con nuevos estatutos que reglamentará la organización representativa, quedaron con llamarla CODECO... La historia de lucha por la tierra se sostuvo, CODECO es una Organización que insta a la formación política, Derechos Humanos y que las personas puedan ser instruidas y puedan conocer sus derechos. (Entrevista a referente)

La CODECO empezó a insertarse en las comunidades, a llevar talleres, capacitaciones en lo que es derecho comunal y derecho del ser humano, lo que el Estado debe velar como derecho. "La CODECO dejó huellas porque hoy en día las personas ya se manifiestan, salen a las calles, las personas ya conocen sus derechos, la gente ya reclama lo que les corresponde, antes no existía eso".

\subsection{Participación de las mujeres}

La lucha de las mujeres en la sociedad capitalista tiene una larga historia, luchas por la igualdad política, legal, profesional que les ha sido negada, bajo opresiones que el patriarcado ejercía y sigue ejerciendo sobre ellas, en este sentido la entrevistada menciona reivindicaciones logradas por las mujeres organizadas en el bañado norte, como emprendimientos para lograr autonomía económica, emancipación y por sobre todo conocer sus derechos como sujeto.

Sabemos que las mujeres están enmarcadas por la lucha por lograr su emancipación, todas las reivindicaciones han sido bajo represiones de carácter arbitrario, en nuestro país la falta de respuesta por parte de las instituciones encargadas en caso de violencia hacia la mujer expone los procesos para actuar en esos casos desordenados y complicados.

Un ejemplo de ello fue el hallazgo en el bañado Norte desde la organización CODECO articulado con Serpaj, han realizado censos, se realizó uno hacia la zona del Mbigua, un lugar con poco acceso, 
“'donde se descubrió que vivían mujeres de 15 a 16 años con cónyuges mucho mayores, las mujeres tenían entre 3 a 4 hijos cada una, en total estado de abandono, se llevó talleres y capacitación a estas mujeres quienes solo pudieron participar un par de veces, no se pudo profundizar por obstáculos, como la falta de no contar con un lugar donde dejar a sus hijos, también porque sus cónyuges no le permitían la participación en las capacitaciones y charlas" (Entrevista a referente).

Según expresan en la Comunidad las personas se encuentran en situación de pobreza y exclusión, olvidados como sujetos de derechos, pero en constante luchas donde las mujeres son protagonistas fundamentales, generalmente lideran la organización para el traslado de las personas en los refugios asignados en tiempos de inundación, preparando las carpas y ollas populares con pocos ingredientes, pero para paliar el hambre a muchos.

CODECO ha sido liderado por mujeres, en un momento al producirse la separación de muchos miembros del movimiento, mayoritariamente las mujeres tomaron la posta de la misma. La lucha en la organización siempre fue más de mujeres.

La entrevistada refiere que han logrado espacios como lideresas dentro de la organización y la comunidad, "Siempre hubo hombres, pero, siempre lideramos las mujeres, en algún momento el coordinador fue hombre, pero más adelante eso terminó ya nadie quiere que un hombre sea coordinador, y tuvimos ese espacio, tenemos trabajando el espacio de las mujeres" (Entrevista a referente).

Existieron reivindicaciones por las mujeres organizadas en el bañador norte, como emprendimientos para lograr la autonomía económica, la emancipación y por sobre todo conocer sus derechos como sujetos.

Las mujeres del bañado son fundamentales en las luchas de las distintas situaciones que se presentan en los territorios a las cuales pertenecen.

\subsection{Derecho a ser libres como mujeres}

En el bañado norte las mujeres son protagonistas en las luchas por el territorio, en las luchas por los derechos de cada ciudadano como ser humano, la CODECO empezó a insertarse en las comunidades llevando talleres, capacitaciones, desnaturalizando la violencia contra las mujeres, trabajando con el conocimiento de los derechos comunales y humanos, dando a conocer a estas personas donde recurrir para exigir sus derechos como ciudadanos, pero aun así falta respuesta por parte de las instituciones encargadas en caso de violencia hacia la mujer, pues los procesos para actuar en estos casos son desordenados y complicados. En el barrio no se cuenta con un lugar para recurrir en esos casos, al recurrir a la comisaría se lo deriva a otro lugar y así el proceso para presentar una denuncia es muy difícil, con mecanismos desordenados y que no son para nada claros (Entrevista a referente).

La coordinación hace tiempo se encuentra al mando de mujeres, tienen varias etapas de sus vidas en este proceso. La CODECO llevó talleres y capacitación a las mujeres del bañado norte, no se pudo profundizar por obstáculos como no tener donde dejar a los hijos o porque los cónyuges ya no les permitía asistir, pero también existen mujeres que han logrado la autonomía económica después de estos talleres, han conocido sus derechos y hoy día ya no sufren de maltratos de ninguna índole.

Así como también han logrado organizar una red de mujeres desde donde trabajaron por las situaciones de las mujeres de vulnerabilidad como madres adolescentes, madres solteras y mujeres que sufrían violencia intrafamiliar, logrando en varios casos erradicar la violencia intrafamiliar y direccionar a las madres adolescentes y solteras para lograr una vida más digna como persona y sobre todo sujeto de derechos.

Las mujeres crearon un movimiento social a partir del cuestionamiento al orden 
de géneros con predominio masculino que se manifiesta concretamente en estructuras, ideologías, instituciones, tradiciones, rituales y leyes que sobrevalorany sancionan el poder de los hombres sobre las mujeres" (Sau, 1990).

\section{Conclusión}

Paraguay es unos de los países más desiguales en tenencias de tierras, con poca o nula voluntad política para garantizar el derecho de la vivienda digna a las personas más vulnerables, lo cual desemboca en invasiones, asentamientos entre otras formas de vivencia informal, situación que se puede ver en el bañado norte. Los pobladores del bañado norte se encuentran en constantes luchas y resistencias por conservar sus viviendas donde la mayoría viven hace más de 15 y 20 años, según los mismos.

La participación de las mujeres en las luchas comunitarias por el territorio en el bañado norte desde la organización CODECO, ayuda a vislumbrar los obstáculos formales e informales, los desafíos y las posibles estrategias para promover la participación de las mujeres en igualdad de condiciones con los hombres en la arena política.

La mujer hoy es el pilar de la lucha social, en el bañado norte, es un factor importante en la lucha por la tierra, es la que actúa en situaciones de necesidad, es quien pone la voz por los derechos. (Entrevista a referente).

Otra de las matrices importantes en esta organización es que las mujeres lograron hacerse parte de la dinámica de vida en el territorio, no solo por el derecho a la tierra sino también su reivindicación dentro de la sociedad como sujeto de derecho, se reconoce el empoderamiento económico de la mujer como necesidad para su bienestar individual y su entorno, es un proceso que contribuye al buen funcionamiento de su salud física, mental y sobre todo en su desarrollo como ser social, autores como Ghuman (2001), Kabeer (1998), Roa (1997) sostienen que el Empoderamiento económico de las mujeres las protege de la violencia de género, lo que las ampara y ayuda a preservar su bienestar en las distintas esferas de la sociedad, en éste sentido las mujeres del bañado norte en su mayoría tienen dependencia económica de los cónyuges, así como también la cantidad de hijos que tienen por falta de educación sexual son obstáculos para ese Empoderamiento, a raíz del cual también sufren violencia física, la fuerza del patriarcado hace creer a las mujeres natural la opresión y subordinación ante su contraparte.

\section{Referencia}

Castel, R. (1995). Les métamorphoses de la question sociale. Fayard.

Houdin, C. (2019). Feminismo de Clase, Utopía o Realidad. https:// clas s room.google.com/u/1/w/ MTExMjl4NzU5OTU5/t/all

Iamamoto, M. (2003). El Servivio Social en la Contemporaneidad. Cortez Editorial.

Palau, M. (2017). Movimientos Sociales: sus demandas en la transición. (El caso de los movimientos de Trabajadores $y$ de Mujeres). CLACSO Consejo Latinoamericano de Ciencias Sociales.

Pleyers, G. (2018). Movimientos sociales en el siglo XXI: perspectivas $y$ herramientas analíticas. CLACSO Consejo Latinoamericano de Ciencias Sociales.

Touraine, A. (2000). ¿Podremos vivir juntos? Iguales y diferentes. Fondo de Cultura Económica. 


\section{Sobre los Autores}

María Graciela Ayala de Villalba

Estudiante de tercer año de la carrera de Trabajo Social en la Facultad de Ciencias Sociales de la Universidad Nacional de Asunción.

\section{José Albino Soto Fernández}

Estudiante de tercer año de la carrera de Trabajo Social en la Facultad de Ciencias Sociales de la Universidad Nacional de Asunción.

Jessica Graciela Castiñeira Giménez

Estudiante de tercer año de la carrera de Trabajo Social en la Facultad de Ciencias Sociales de la Universidad Nacional de Asunción.

\section{Mercedes Ríos}

Estudiante de tercer año de la carrera de Trabajo Social en la Facultad de Ciencias Sociales de la Universidad Nacional de Asunción.

\section{Rosa Ruffinelli}

Docente de Abordaje Profesional II de la carrera de Trabajo Social en la Facultad de Ciencias Sociales de la Universidad Nacional de Asunción. 